\title{
Regulation of TLR4 expression mediates the attenuating effect of erythropoietin on inflammation and myocardial fibrosis in rat heart
}

\author{
FEI LIU ${ }^{1 *}$, YUAN WEN ${ }^{2 *}$, JINYUAN KANG ${ }^{3}$, CHUNYING WEI $^{2}$, \\ MENGHONG WANG ${ }^{2}$, ZEQI ZHENG ${ }^{2}$ and JINGTIAN PENG ${ }^{2}$ \\ Departments of ${ }^{1}$ Obstetrics and Gynecology, and ${ }^{2}$ Cardiovascular Medicine, The First Affiliated Hospital of \\ Nanchang University, Nanchang, Jiangxi 330006; ${ }^{3}$ Department of Cardiovascular Medicine, \\ The Third Hospital of Nanchang, Nanchang, Jiangxi 330009, P.R. China
}

Received November 1, 2017; Accepted May 4, 2018

DOI: $10.3892 /$ ijmm.2018.3707

\begin{abstract}
The mechanism underlying the anti-inflammatory or antifibrotic activity of erythropoietin (EPO) in myocardial fibrosis (MF) remains elusive. In the current study, abdominal aortic constriction (AAC) was performed on rats and EPO and/or Toll-like receptor (TLR)4 were overexpressed in rat hearts through intramyocardial administration of lentivirus expressing the EPO and TLR4 genes. Hematoxylin and eosin staining and Masson's trichrome staining were performed on tissue sections from rat hearts for histopathological examination. ELISA was used to determine the levels of inflammatory mediators in serum. Gene expression levels were determined by quantitative polymerase chain reaction analysis and protein expression levels were determined by western blot analysis and immunofluorescence staining. The results indicated that EPO overexpression improved MF in rat hearts, by inhibiting the release of transforming growth factor (TGF)- $\beta 1$, tumor necrosis factor (TNF)- $\alpha$, interleukin (IL)-6, IL-1 $\beta$, IL-17A, matrix metalloproteinase (MMP)-9 and MMP-2. Moreover, EPO overexpression suppressed the expression of TLR4, while promoting phosphoinositide 3-kinase (PI3K) and phosphorylated AKT serine/threonine kinase 1 (Akt) expression levels. However, the beneficial effects of EPO were attenuated by overexpression of TLR4. In addition, inhibition of PI3K/Akt signaling activity by treatment with LY294002 markedly reversed the protective effect of EPO on the AAC-induced MF. Taken together, the
\end{abstract}

Correspondence to: Dr Yuan Wen, Department of Cardiovascular Medicine, The First Affiliated Hospital of Nanchang University, 17 Yongwaizheng Street, Nanchang, Jiangxi 330006, P.R. China

E-mail: ywenjx@163.com

*Contributed equally

Key words: erythropoietin, inflammatory factors, myocardial fibrosis, phosphoinositide 3-kinase, AKT serine/threonine kinase, Toll-like receptor 4 present study demonstrated that EPO may have a critical role against MF by activating PI3K/Akt signaling and by downregulating TLR4 expression, thereby inhibiting the release of TGF- $\beta 1$, TNF- $\alpha$, IL-6, IL-1 $\beta$, IL-17A, MMP-9 and MMP-2. These findings suggest that the PI3K/Akt/TLR4 signaling pathway is associated with the anti-inflammatory effects of EPO and may play a role in attenuating AAC-induced MF.

\section{Introduction}

As one of the major global health concerns, myocardial fibrosis (MF) is closely associated with almost all types of heart disease (1). Excessive fibroblast accumulation is the direct cause of MF in hearts that undergo pressure overload, which has been a major clinical issue for physicians in the clinic (2). Accordingly, there is an unmet need for identification of novel therapeutic strategies towards attenuating MF in the cardiovascular field.

Erythropoietin (EPO) is synthesized by the kidney and the liver in fetal life as a hematopoietic hormone, and directly participates in mammalian erythropoiesis (3). Previous studies have demonstrated that EPO has significant protective effects on the heart (4), kidney (5), or nerves (6), and inhibits inflammatory effects (7). As a multifunctional molecule and because its receptors are widely expressed by various organs, EPO promotes organ regeneration and improves fibrosis $(8,9)$. In our preliminary study, EPO had significant anti-inflammatory effects and attenuated fibrosis in a pressure-overload rat model that was subjected to abdominal aortic constriction (AAC) (10). Furthermore, it has been indicated that EPO may be a powerful cardioprotective agent and a potential component for antifibrotic therapies (11-13). The protective mechanism may be via inhibiting the secretion of inflammatory factors, including transforming growth factor (TGF) $\beta$, the interleukin (IL) family, and tumor necrosis factors (TNFs) $(11,14)$. However, the specific mechanism underlying the anti-inflammatory or antifibrotic activity of EPO in MF remains elusive. The present study was designed to investigate the regulatory role of EPO in rat MF and to determine whether EPO participates in the attenuation of MF. 


\section{Materials and methods}

Reagents. LY294002 was purchased from Selleckchem (Houston, TX, USA). Antibodies targeting phosphoinositide 3-kinase (PI3K) (cat. no. 21890-1-AP, working dilution: 1:500), AKT serine/threonine kinase 1 (Akt) (catalog number: 10176-2-AP, working dilution: 1:500), phosphorylated (p-) Akt (cat. no. 66444-1-Ig, working dilution: 1:1,000), Toll-like receptor 4 (TLR4) (catalog number: 19811-1-AP, working dilution: 1:500), TGF- $\beta 1$ (cat. no. 21898-1-AP, working dilution: 1:500), matrix metalloproteinase (MMP)-2 (cat. no. 10373-2-AP, working dilution: 1:500) and MMP-9 (cat. no. 10375-2-AP, working dilution: 1:500) were acquired from ProteinTech Group, Inc. (Chicago, IL, USA). The antibody targeting $\beta$-actin (cat. no. KL002; 1:500) was purchased from Jiancheng Bioengineering Institute of Nanjing (Nanjing, China), and the goat anti-mouse Immunoglobulin G (cat. no. SA00001-1, working dilution: 1:2,000) and Goat anti-rabbit IgG (cat. no. SA00001-2, working dilution: 1:2,000) horseradish peroxidase (HRP)-conjugated secondary antibodies were from ProteinTech Group, Inc. ELISA kits for the detection of TGF- $\beta 1$ (cat. no. ELR-TGFb1-1), IL-1 $\beta$ (cat. no. ELR-IL1b-1), IL-6 (cat. no. ELR-IL6-1), IL-17A, and TNF- $\alpha$ (cat. no. ELR-TNFa-1) were purchased from RayBiotech, Inc. (Atlanta, GA, USA).

Animals. Adult male Sprague Dawley rats (age, 6 weeks; weight, 250-300 g) were obtained from the Nanchang University Laboratory Animal Center (Nanchang, China) and were maintained under a 12 -h dark/light cycle at a temperature of $22-25^{\circ} \mathrm{C}$. Animals were allowed access to food and water ad libitum. Experiments involving animals were performed according to the Guide for the Care and Use of Laboratory Animals (National Institutes of Health, Bethesda, MD, USA), and approved by the Experimental Animal Ethics Committee of the Nanchang University (Nanchang, China; Approval no. 2015050612).

Construction of recombinant adenovirus for expression of $E P O$ and TLR4. Using site-specific recombination cloning, EPO (accession no. NM_017001) was cloned into the GV287 vector, (Shanghai Genechem Co., Ltd., Shanghai, China), with a sequence element of Ubi-MCS-3FLAG-SV40-EGFP. TLR4 (accession no. NM_019178) was cloned into the GV320 vector (Shanghai Genechem Co., Ltd.) and had a sequence element of Ubi-MCS-3FLAG-SV40-Cherry. Plasmids containing target genes were transfected into 293T cells using envelope and packaging plasmids. The supernatant was harvested following density gradient centrifugation and stored at $-80^{\circ} \mathrm{C}$. The tissue culture infection dose was used to determine the virus titer.

Experimental groups and treatments. Rats were randomly divided into 5 groups ( $\mathrm{n}=6$ per group), as follows: Control group, AAC only group, AAC with EPO overexpression (AAC + EPO) group, AAC with EPO and TLR4 co-overexpression (AAC + EPO + TLR4) group, and AAC with EPO overexpression combined with LY294002 administration $(\mathrm{AAC}+\mathrm{EPO}+\mathrm{LY} 294002)$ group. Rats in the AAC group were subjected to an abdominal aortic banding procedure following anesthesia with intraperitoneal (i.p.) injection of chloral hydrate $(350 \mathrm{mg} / \mathrm{kg})$. In brief, rats were anesthetized with $2 \%$ chloral hydrate $(2 \mathrm{ml} / \mathrm{kg})$ and the abdominal aorta was exposed by a midline abdominal incision. The aorta was ligated against a 22-Gauge blunted needle using silk sutures (5-0). Then, the needle was rapidly retracted to create a defined constriction (15). Rats in the AAC + EPO group underwent $\mathrm{AAC}$ and the EPO gene was directly transferred into the myocardium by intramyocardial administration of $2 \times 10^{7}$ transduction units (TU) EPO-recombinant lentivirus. For the intramyocardial administration, a thoracotomy was performed on the anesthetized rat, and then the myocardium was injected at multiple points with lentivirus using a syringe with a fine needle tip (16). Rats in the AAC + EPO + TLR4 group were subjected to AAC, and then EPO and TLR4-expressing lentivirus was simultaneously injected into the myocardium by intramyocardial administration with $2 \times 10^{7} \mathrm{TU}$ lentivirus particles. Successful overexpression of EPO and TLR4 in the heart tissue was confirmed by microscopy (Fig. 1). Rats in the AAC + EPO + LY294002 group underwent surgery similar to rats in the AAC + EPO group, and in addition they were i.p. administered with $5 \mathrm{mg} / \mathrm{kg} /$ day LY294002 as continuous intervention for 4 weeks. The time point and doses of LY294002 used in the current experiments were determined during preliminary experiments. Rats in the control group underwent sham surgery. After 8 weeks, all rats were sacrificed under anesthesia using an i.p. injection of chloral hydrate $(350 \mathrm{mg} / \mathrm{kg})$, following which blood was harvested through the abdominal aorta to prepare serum and the hearts were collected. Left ventricles were dissected, weighed and divided by body weight to calculate the left ventricular weight index (LVWI). All samples were stored at $-80^{\circ} \mathrm{C}$.

Histopathological examination. Histopathological examination was performed as described in previous studies (17). In brief, rat hearts were dissected, fixed in $4 \%$ paraformaldehyde at room temperature for $\geq 12 \mathrm{~h}$, dehydrated in 70, 80, 90, 95 and $100 \%$ ethanol, vitrified with xylene, embedded in paraffin $\left(60-62^{\circ} \mathrm{C}\right)$, and sectioned using a microtome (Thermo Fisher Scientific, Inc., Waltham, MA, USA) into $4 \mu \mathrm{m}$ thick sections. Sections were then subjected to hematoxylin and eosin (H\&E) staining or Masson's trichrome staining and visualized using an optical light microscope (Olympus Corporation, Tokyo, Japan). For Masson's trichrome staining, sections were deparaffinized and embedded in a Masson composition solution, following the manufacturer's guidelines, to determine changes in interstitial fibrosis. A total of 6 discontinuous sections per specimen and 5 fields per section were selected for analysis to determine the total collagen content and \% of fibrotic area using Image Pro Plus 6.0 software (National Institutes of Health).

Serum analysis. In order to evaluate the levels of TGF- $\beta 1$, IL-1 $\beta$, IL-6, IL-17A, and TNF- $\alpha$ in rat serum, ELISA was performed according to the manufacturer's guidelines (RayBiotech, Inc.).

Reverse transcription-quantitative polymerase chain reaction (RT-qPCR) assay. Total RNA was extracted from frozen myocardial tissue using TRIzol reagent (Thermo Fisher Scientific, Inc.), following the manufacturer's guidelines. Reverse transcription was conducted using Transcriptor First Strand cDNA Synthesis kit (Roche Diagnostics, Indianapolis, IN, USA). Premier Primer 5.0 software (http://www.premierbiosoft.com/primerdesign/) 
was used to design the oligonucleotide primer sequences, as presented in Table I. $\beta$-actin was used as an internal control. The synthesized first-strand cDNA samples were subjected to qPCR using a SYBR Green PCR Master Mix (Toyobo Bio-Technology, Shanghai, China) and the qPCR reaction was performed on an ABI Prism 7700 Sequence Detector (Applied Biosystems; Thermo Fisher Scientific, Inc.). The integrity of PCR products was confirmed by dissociation curve analysis using 7500 Software v2.0 (Applied Biosystems,; Thermo Fisher Scientific, Inc.). Threshold cycle $(\mathrm{Cq})$ values were determined and relative fold changes in mRNA expression were calculated using the formula $2^{-\Delta \Delta \mathrm{Cq}}(18)$.

Western blot analysis. Myocardial tissue was dissociated with a total protein extraction kit (Applygen Technologies, Inc., Beijing, China), according to the manufacturer's instructions and tissue lysates were evaluated by western blot analysis (19). In brief, from each myocardial tissue sample, a total of $30 \mathrm{mg}$ protein was separated by SDS-PAGE (10\% gel) and electrotransferred onto polyvinylidene difluoride membranes. Membranes were blocked in 5\% non-fat milk diluted in Tris-buffered saline/0.1\% Tween-20 (TBST) at room temperature for $2 \mathrm{~h}$. Next, they were probed with primary antibodies (1:500) targeting PI3K, Akt, anti-p-Akt, TLR4, TGF- $\beta 1$, MMP-2, MMP-9 and anti- $\beta$-actin diluted in 5\% non-fat milk at $4^{\circ} \mathrm{C}$ overnight. Following three washes with TBST for $15 \mathrm{~min}$ at room temperature, the membranes were incubated with HRP-conjugated secondary antibodies (anti-rabbit IgG or anti-rat $\mathrm{IgG}$ ) for $2 \mathrm{~h}$ at room temperature. The membranes were
Table I. Primers used in quantitative polymerase chain reaction analysis.

\begin{tabular}{|c|c|}
\hline Gene & Primer sequence $\left(5^{\prime}-3^{\prime}\right)$ \\
\hline$\beta$-actin & $\begin{array}{l}\text { Forward: CTGGGACGACATGGAGAAAA } \\
\text { Reverse: AAGGAAGGCTGGAAGAGTGC }\end{array}$ \\
\hline TLR4 & $\begin{array}{l}\text { Forward: TGGCATCATCTTCATTGTCC } \\
\text { Reverse: CAGAGCATTGTCCTCCCACT }\end{array}$ \\
\hline PI3K & $\begin{array}{l}\text { Forward: ATAATGCTGGGGAGGATGC } \\
\text { Reverse: TAGGGTGTCGCTGTGGAAAT }\end{array}$ \\
\hline Akt1 & $\begin{array}{l}\text { Forward: ACTCATTCCAGACCCACGAC } \\
\text { Reverse: ACACAATCTCCGCACCGTA }\end{array}$ \\
\hline
\end{tabular}

TLR4, Toll-like receptor 4; PI3K, phosphoinositide 3-kinase; Akt1, AKT serine/threonine kinase 1 .

washed with TBST three times for $20 \mathrm{~min}$ at room temperature. Immune complexes were visualized by an enhanced chemiluminescence (ECL) method and band intensities were evaluated quantitatively using the Quantity One software (Bio-Rad Laboratories, Inc., Hercules, CA, USA).

Statistical analysis. Data are presented as the mean \pm standard error of the mean from six independent experiments. Results were evaluated by a one-way analysis of variance with a Student-Newman-Keuls q test as a post-hoc, performed using
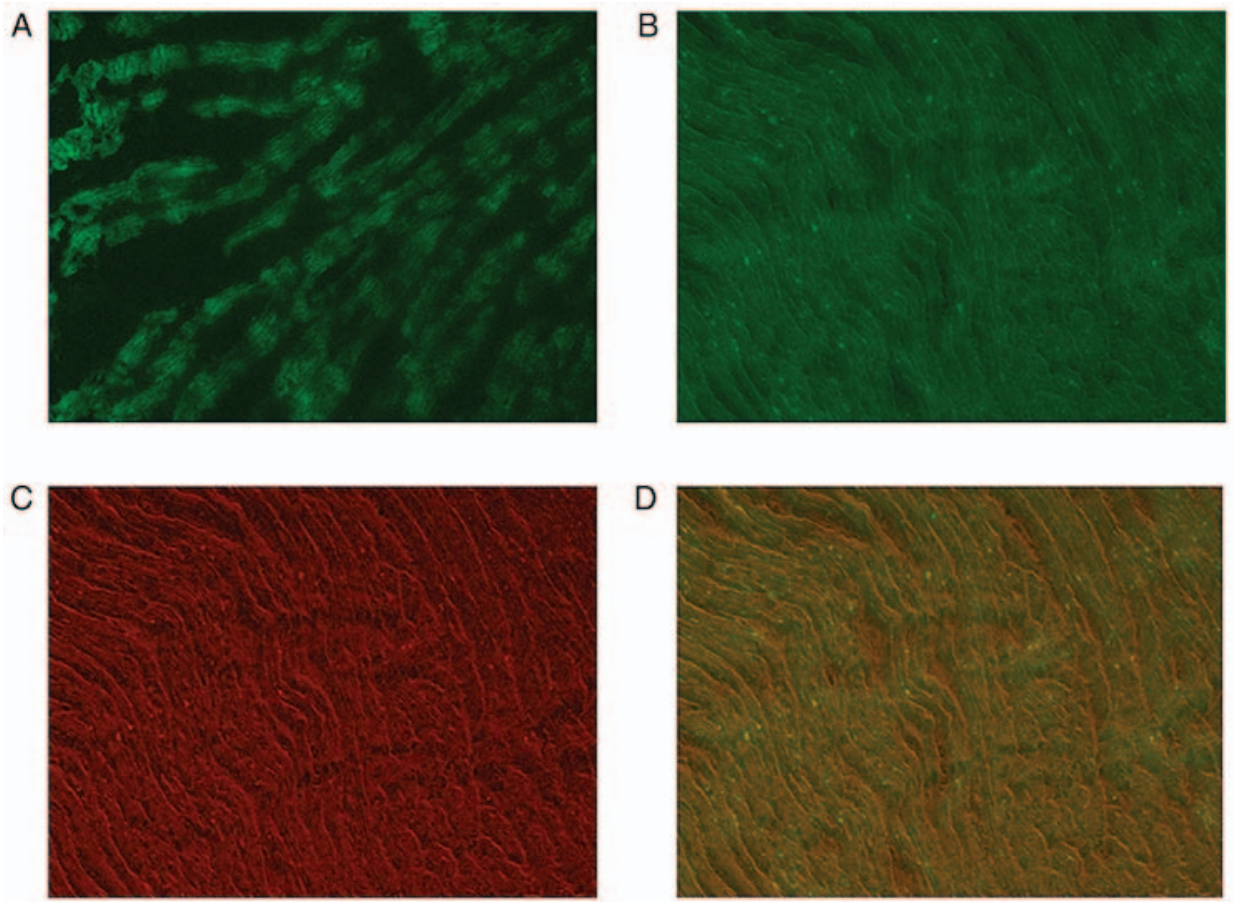

Figure 1. Target gene expression in left ventricular wall. Microscopy analysis was performed in order to determine whether the target genes were stably expressed following intramyocardial administration of recombinant lentivirus for 1 month. The left ventricular wall of randomly selected rats was stripped, frozen, sectioned by a microtome, and visualized using immunofluorescence analysis. (A) EPO expression in the left ventricular wall of AAC rats, injected with EPO-expressing lentivirus (magnification, x200). The sequence element contained EGFP (green signal). EPO displayed a homogenous expression in the entire left ventricular wall. (B) EPO expression levels in the left ventricular wall of AAC rats treated with EPO + TLR4 (magnification, x200). (C) TLR4 expression levels in the left ventricular wall of AAC rats treated with EPO + TLR4. The sequence element of the TLR4-expressing lentiviral vector contained cherry fluorescent protein (magnification, x200; red signal). (D) Merged image of B and C. EPO and TLR4 demonstrated a homogenous expression in the left ventricular wall. EPO, erythropoietin; AAC, abdominal aortic constriction; EGFP, enhanced green fluorescent protein; TLR4, Toll-like receptor 4. 
Table II. LVWI in the rat experimental groups.

\begin{tabular}{llll}
\hline Experimental group & $\mathrm{N}$ & $\mathrm{BW}(\mathrm{g})$ & LVWI $(\mathrm{mg} / \mathrm{g})$ \\
\hline Control & 6 & $290.22 \pm 16.09$ & $2.11 \pm 0.09$ \\
AAC & 6 & $277.35 \pm 18.56$ & $2.79 \pm 0.22^{\mathrm{a}}$ \\
AAC + EPO & 6 & $289.46 \pm 16.33$ & $2.28 \pm 0.17^{\mathrm{b}}$ \\
AAC + EPO + TLR4 & 6 & $279.89 \pm 19.44$ & $2.66 \pm 0.20^{\mathrm{c}}$ \\
AAC + EPO+ LY294002 & 6 & $284.76 \pm 17.23$ & $2.58 \pm 0.23^{\mathrm{c}}$ \\
\hline
\end{tabular}

The LVWI was calculated according to the formula: $\mathrm{LVWI}=\mathrm{LVW}(\mathrm{mg}) / \mathrm{BW}(\mathrm{g})$. ${ }^{\mathrm{a}} \mathrm{P}<0.05 \mathrm{vs}$. control group; ${ }^{\mathrm{b}} \mathrm{P}<0.05 \mathrm{vs}$. AAC group; and ${ }^{\mathrm{c}} \mathrm{P}<0.05$ vs. AAC + EPO group. LVWI, left ventricular weight index; BW, body weight; LVW, left ventricle weight; AAC, abdominal aortic constriction; EPO, erythropoietin; TLR4, Toll-like receptor 4.

SPSS software (version 20.0; IBM Corp., Armonk, NY, USA). $\mathrm{P}<0.05$ was considered statistically significant.

\section{Results}

EPO overexpression improves cardiac hypertrophy in rats. The level of cardiac hypertrophy in rat hearts was assessed by evaluating the LVWI. As presented in Table II, significant increases in LVWI were observed in the AAC group compared with the control group $(\mathrm{P}<0.05)$. Treatment with EPO significantly decreased the LVWI compared with the ACC group alone $(\mathrm{P}<0.05$; Table II). However, the effect of EPO was significantly reversed by TLR4 overexpression in the $\mathrm{AAC}+\mathrm{EPO}+\mathrm{TLR} 4$ group, and by PI3K inhibition in the $\mathrm{AAC}+\mathrm{EPO}+\mathrm{LY} 294002$ group $(\mathrm{P}<0.05$; Table II $)$.

EPO overexpression improves myocardial fibrosis in rats. $\mathrm{H} \& \mathrm{E}$ staining was performed to evaluate pathological changes in the rat myocardium in each treatment group (Fig. 2A). In the control group, the morphology and structure of the cells and their nuclei was normal. By contrast, in the AAC group, the cardiomyocytes were hyperplastic, the intercellular substance was expanded, and the myofibrils displayed a disrupted and disordered arrangement. In the AAC + EPO group, the morphology of the myocardium was significantly improved. Myocardial fibers were contiguously and more neatly arranged, and the morphology and structure of the cells and their nuclei had a normal appearance. However, the effects of EPO were significantly reversed by treatment with TLR4 or LY294002.

Masson's trichrome staining was performed to determine the degree of MF in rat hearts. Histological analysis revealed an increase of collagen fibers and disordered arrangement of cardiac muscle fibers in the AAC only group when compared to control, which was significantly improved by treatment with $\mathrm{EPO}$ in the AAC + EPO group (Fig. 2B and C). However, the effects of $\mathrm{EPO}$ on MF were reversed in the $\mathrm{AAC}+\mathrm{EPO}+$ TLR4 and AAC + EPO + LY294002 groups (Fig. 2B and C).

EPO overexpression suppresses the secretion of inflammatory factors. ELISA was used to determine the levels of TGF- $\beta 1$, TNF- $\alpha$, IL-1 $\beta$, IL-6 and IL-17A in rat serum. As presented in Table III and Fig. 3, AAC increased the secreted levels of TGF- $\beta 1$, TNF- $\alpha$, IL- $1 \beta$, IL- 6 and IL-17A in the serum compared with the control group $(\mathrm{P}<0.05)$. The expression of
A
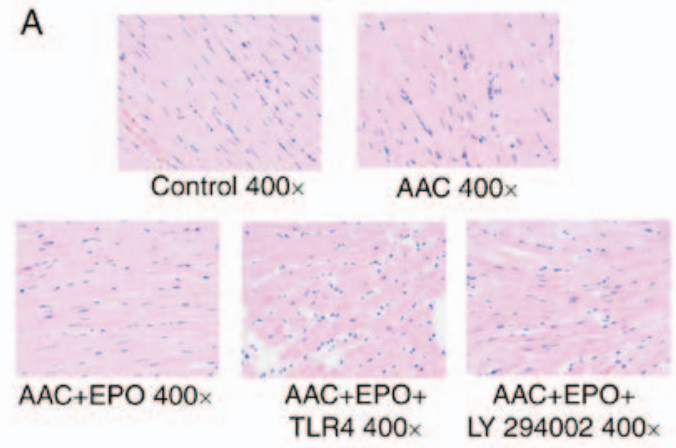

B

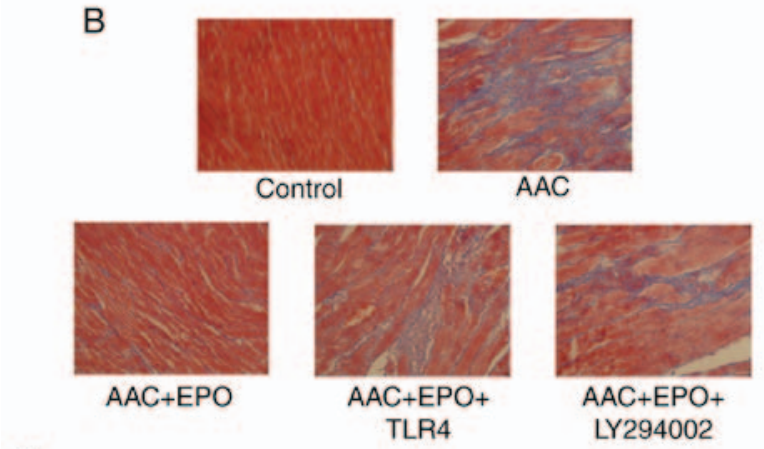

C

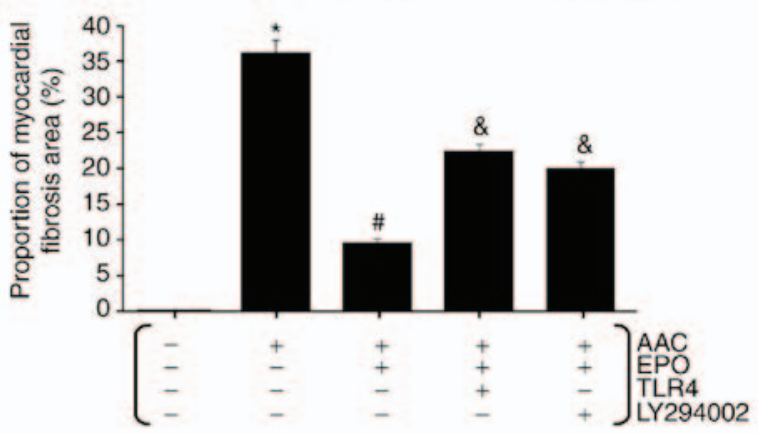

Figure 2. Histopathological analysis of the heart tissue in each experiment group. (A) Representative images of H\&E staining. (B) Representative images of Masson's trichrome staining. (C) Quantification of the proportion of myocardial fibrosis area using Masson's trichrome staining. The data are expressed as the mean \pm standard error of the mean ( $n=6$ per group). ${ }^{*} \mathrm{P}<0.05$ vs. the control group; ${ }^{\#} \mathrm{P}<0.05$ vs. the AAC only group; and ${ }^{\circledR} \mathrm{P}<0.05$ vs. the $\mathrm{AAC}+\mathrm{EPO}$ group. H\&E, hematoxylin and eosin; AAC, abdominal aortic constriction; EPO, erythropoietin; TLR4, Toll-like receptor 4.

inflammatory mediators was reduced in rats in the AAC + EPO group compared with the AAC only group (Fig. 3; Table III). 
Table III. Concentrations of TGF- $\beta 1$, TNF- $\alpha$, IL-1 $\beta$, IL-6 and IL-17A in rat serum.

\begin{tabular}{|c|c|c|c|c|c|}
\hline Experimental group & TGF- $\beta 1$ (ng/l) & TNF- $\alpha(n g / l)$ & IL-1 $\beta(n g / l)$ & IL-6 (ng/l) & IL-17A (ng/l) \\
\hline Control & $3,912.62 \pm 108.98$ & $252.17 \pm 28.40$ & $7.91 \pm 0.66$ & $43.78 \pm 3.66$ & $5.69 \pm 0.65$ \\
\hline $\mathrm{AAC}$ & $8,895.42 \pm 203.58$ & $426.54 \pm 47.28^{\mathrm{a}}$ & $34.85 \pm 3.70^{\mathrm{a}}$ & $156.46 \pm 10.26^{\mathrm{a}}$ & $25.92 \pm 1.67^{\mathrm{a}}$ \\
\hline $\mathrm{AAC}+\mathrm{EPO}$ & $5,241.12 \pm 167.21$ & $274.99 \pm 37.02^{\mathrm{b}}$ & $15.82 \pm 2.45^{\mathrm{b}}$ & $68.22 \pm 4.07^{\mathrm{b}}$ & $8.40 \pm 1.45^{\mathrm{b}}$ \\
\hline $\mathrm{AAC}+\mathrm{EPO}+\mathrm{TLR} 4$ & $7,192.57 \pm 199.67$ & $363.13 \pm 31.45^{\mathrm{c}}$ & $30.64 \pm 1.45^{\mathrm{c}}$ & $108.25 \pm 7.50^{c}$ & $18.70 \pm 1.05^{\mathrm{c}}$ \\
\hline $\mathrm{AAC}+\mathrm{EPO}+\mathrm{LY} 294002$ & $6,980.75 \pm 189.88$ & $328.56 \pm 29.21^{\mathrm{c}}$ & $25.25 \pm 0.56^{\mathrm{c}}$ & $95.48 \pm 6.72^{c}$ & $16.49 \pm 2.56^{\mathrm{c}}$ \\
\hline
\end{tabular}

${ }^{\mathrm{a}} \mathrm{P}<0.05$ vs. control group; ${ }^{\mathrm{b}} \mathrm{P}<0.05 \mathrm{vs}$. AAC group; and ${ }^{\mathrm{c}} \mathrm{P}<0.05 \mathrm{vs}$. AAC + EPO group. TGF, transforming growth factor; TNF, tumor necrosis factor; IL, interleukin; AAC, abdominal aortic constriction; EPO, erythropoietin; TLR4, Toll-like receptor 4.
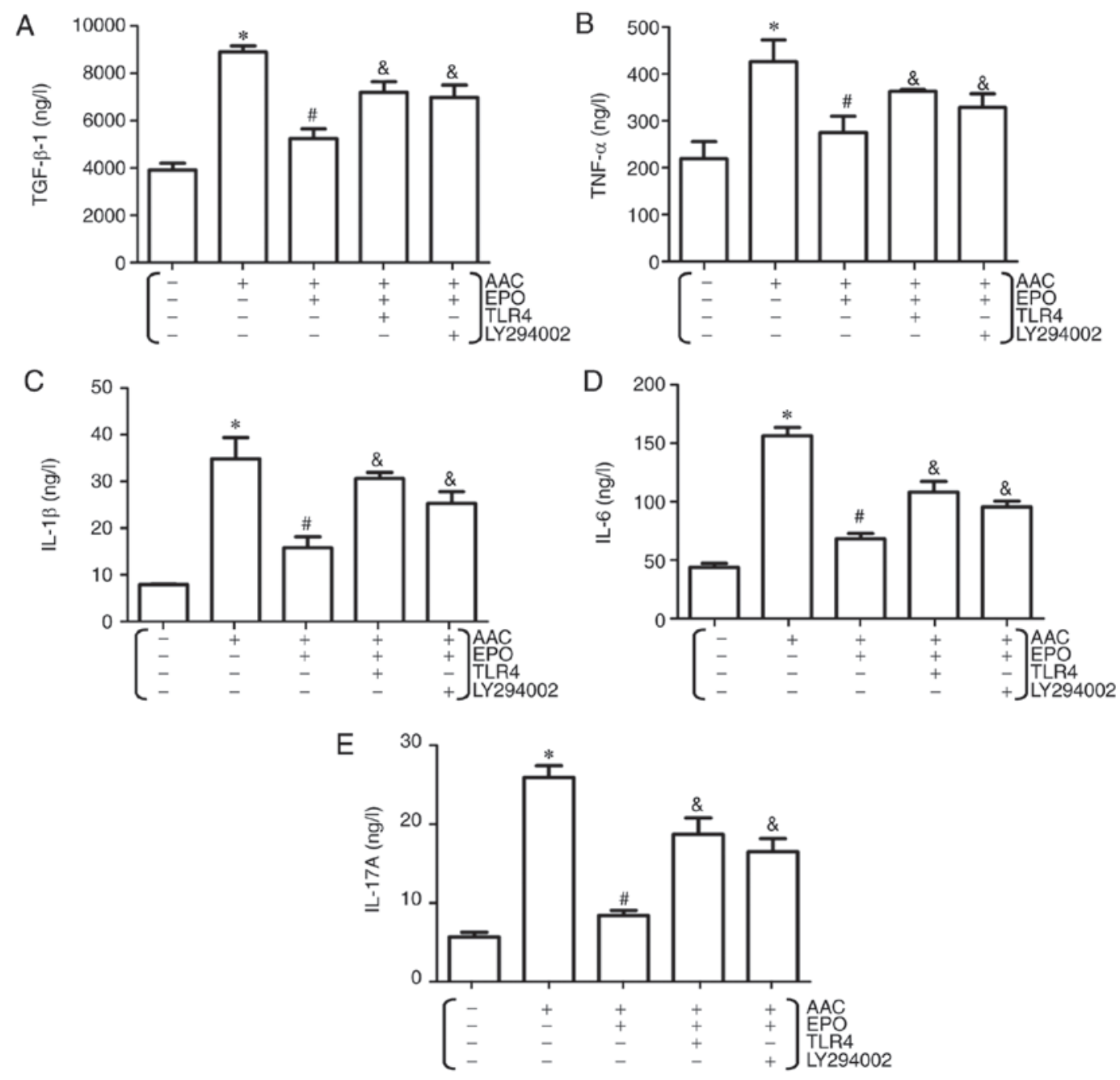

Figure 3. Effects of EPO overexpression on the serum levels of inflammatory mediators in rats that underwent AAC. ELISA was employed to determine the levels of inflammatory mediators (A) TGF- $\beta 1$, (B) TNF- $\alpha$, (C) IL-1 $\beta$, (D) IL-6 and (E) IL-17A in the rat serum in each group. The data are expressed as the mean \pm standard error of the mean ( $\mathrm{n}=6$ per group). ${ }^{*} \mathrm{P}<0.05$ vs. the control group; ${ }^{~} \mathrm{P}<0.05$ vs. the AAC only group; and ${ }^{\&} \mathrm{P}<0.05$ vs. the AAC $+\mathrm{EPO}$ group. EPO, erythropoietin; AAC, abdominal aortic constriction; TGF, transforming growth factor; TNF, tumor necrosis factor; IL, interkleukin; TLR4, Toll-like receptor 4.

By contrast, the effect of EPO on inflammatory factor secretion was significantly reversed in rats in the AAC + EPO + TLR4 or AAC + EPO + LY294002 groups (P<0.05; Fig. 3; Table III).

Gene expression in the different experimental groups. The mRNA expression levels of TLR4, PI3K and Akt in the different groups were evaluated by RT-qPCR. As illustrated in Fig. 4, the expression levels of TLR4 in rats in the AAC group were high, whereas in the AAC + EPO group, the levels were significantly decreased. Notably, in the AAC + EPO + TLR4 and AAC + EPO + LY294002 groups, TLR4 mRNA levels were significantly increased compared with the AAC + EPO group ( $\mathrm{P}<0.05$; Fig. 4). Compared with the control, $\mathrm{PI} 3 \mathrm{~K}$ and Akt1 genes in the AAC only group were poorly expressed, 


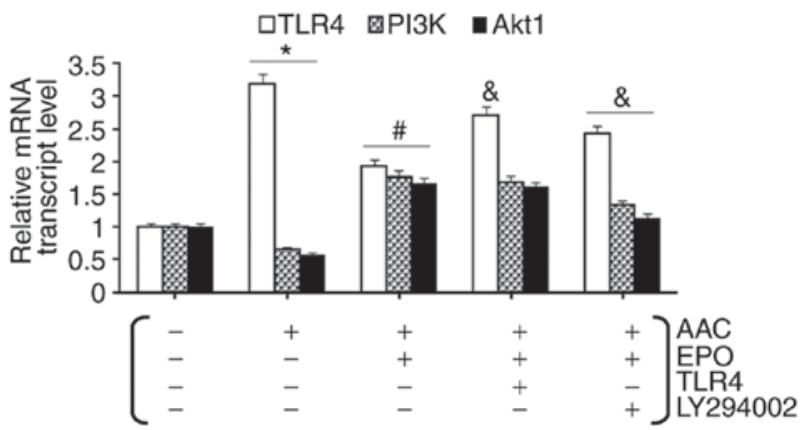

Figure 4. Effects of EPO overexpression on gene expression of TLR4, PI3K and Aktl in different groups. Relative mRNA expression levels were examined in the heart tissues in each group. The data are expressed as the mean \pm standard error of the mean ( $\mathrm{n}=6$ per group). ${ }^{\mathrm{P}}<0.05$ vs. the control group; ${ }^{\#} \mathrm{P}<0.05$ vs. the $\mathrm{AAC}$ only group; and ${ }^{\&} \mathrm{P}<0.05$ vs. the $\mathrm{AAC}+\mathrm{EPO}$ group. $\mathrm{EPO}$, erythropoietin; TLR4, Toll-like receptor 4; PI3K, phosphoinositide 3-kinase; Akt1, AKT serine/threonine kinase 1; AAC, abdominal aortic constriction.

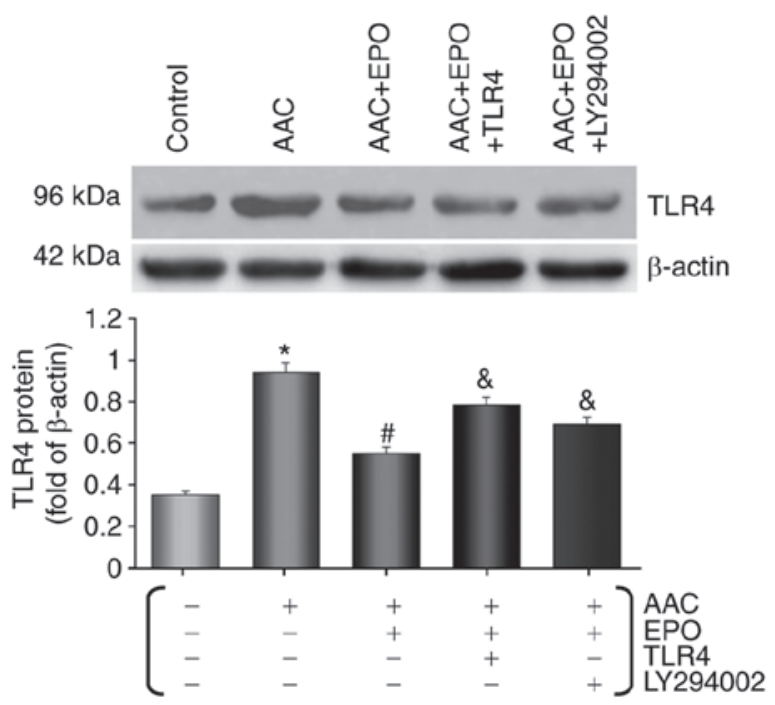

Figure 5. Effects of EPO overexpression on the protein levels of TLR4 in hearts of rats subjected to AAC. The protein expression levels of TLR4 were determined by western blot analysis. The data are expressed the mean \pm standard error of the mean ( $\mathrm{n}=6$ per group). ${ }^{*} \mathrm{P}<0.05$ vs. the control group; ${ }^{*} \mathrm{P}<0.05$ vs. the $\mathrm{AAC}$ only group; and ${ }^{\&} \mathrm{P}<0.05$ vs. the $\mathrm{AAC}+\mathrm{EPO}$ group. $\mathrm{EPO}$, erythropoietin; TLR4, Toll-like receptor 4; AAC, abdominal aortic constriction.

whereas they were significantly upregulated in the $\mathrm{AAC}+\mathrm{EPO}$ group (Fig. 4). In addition, in the AAC + EPO + TLR4 group, expression of PI3K and Akt1 were slightly downregulated compared with the AAC + EPO group, but this change was not significant $(\mathrm{P}>0.05)$. Administration of LY294002 significantly attenuated the effect of EPO on upregulating the expression of PI3K or Akt1 (Fig. 4). These findings indicated a negative association between the levels of TLR4 mRNA and the levels of PI3K and Akt1 mRNAs.

EPO overexpression inhibits TLR4 protein expression, while it increases PI3K and p-Akt expression. To determine the association between TLR4 and PI3K/Akt, TLR4, $\mathrm{PI} 3 \mathrm{~K}, \mathrm{Akt}$, and $\mathrm{p}-\mathrm{Ak} 1$ protein levels in the heart tissues of the experimental rats were evaluated by western blot analysis. As illustrated in Fig. 5, AAC resulted in a significant increase in TLR4 protein expression compared with
A
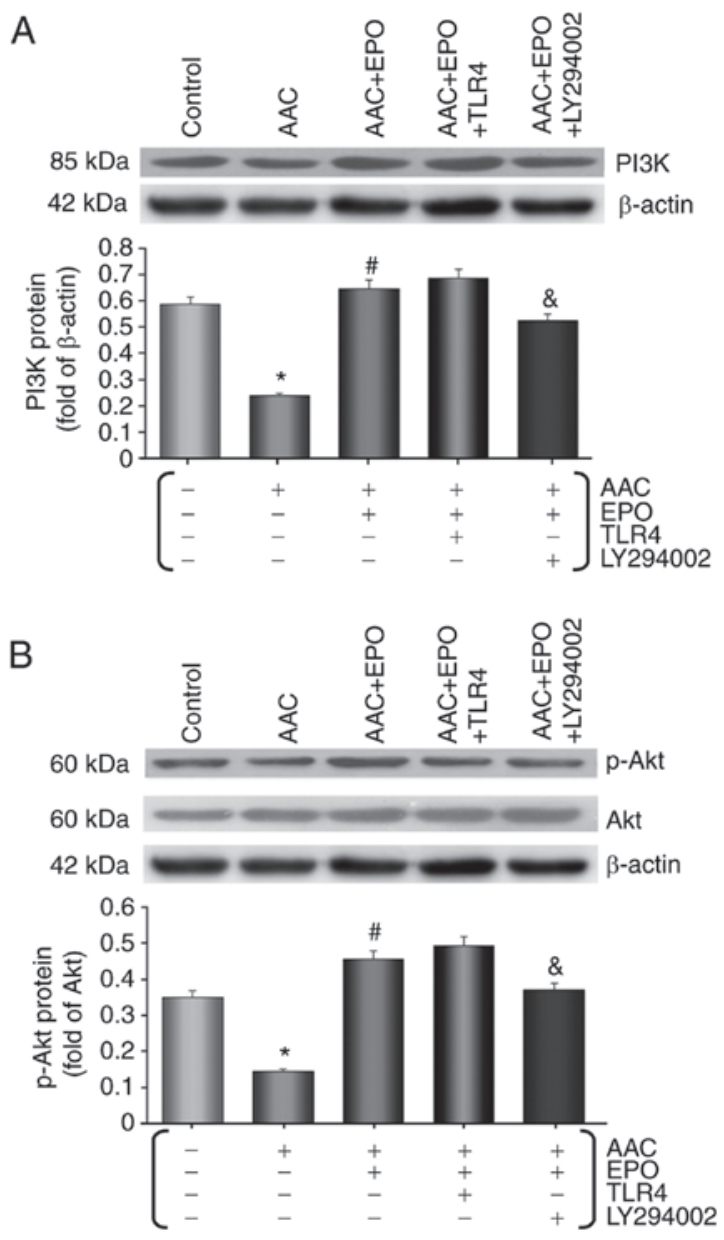

Figure 6. (A and B) Effects of EPO overexpression on the protein levels of PI3K, Akt and p-Akt in hearts of rats subjected to AAC. Western blot analysis was performed to determine the protein levels of PI3K, Akt and p-Akt. The data are expressed the mean \pm standard error of the mean ( $n=6$ per group). $\mathrm{P}<0.05$ vs. the control group; ${ }^{\#} \mathrm{P}<0.05$ vs. the AAC only group; and ${ }^{\circledR} \mathrm{P}<0.05$ vs. the $\mathrm{AAC}+$ EPO group. EPO, erythropoietin; Akt, AKT serine/threonine kinase 1; p-, phosphorylated; AAC, abdominal aortic constriction; TLR4, Toll-like receptor 4.

the control group. EPO treatment significantly decreased the TLR4 protein expression when compared with the AAC only group (Fig. 5). However, in the groups AAC + EPO + TLR4 and AAC + EPO + LY294002, the TLR4 expression levels were significantly upregulated (Fig. 5). By contrast, decreased expression levels of PI3K and p-Akt were observed in the AAC only group compared with the control, whereas in the AAC + EPO group, they were significantly upregulated compared with the AAC only group (Fig. 6). These effects were significantly reduced following administration of LY294002 (Fig. 6).

EPO overexpression suppresses TGF- $\beta 1$ expression. TGF- $\beta 1$ is recognized as a key factor in tissue fibrosis $(9,10)$. To examine whether TGF- $\beta 1$ was activated in AAC-induced MF, the expression levels of TGF- $\beta 1$ were assessed by western blot analysis. As illustrated in Fig. 7, AAC induced a significant increase in the expression of TGF- $\beta 1$. In comparison with the AAC only group, treatment with EPO significantly decreased the TGF- $\beta 1$ expression levels. However, these effects were prevented by TLR4 overexpression or co-administration of LY294002 (Fig. 7). 


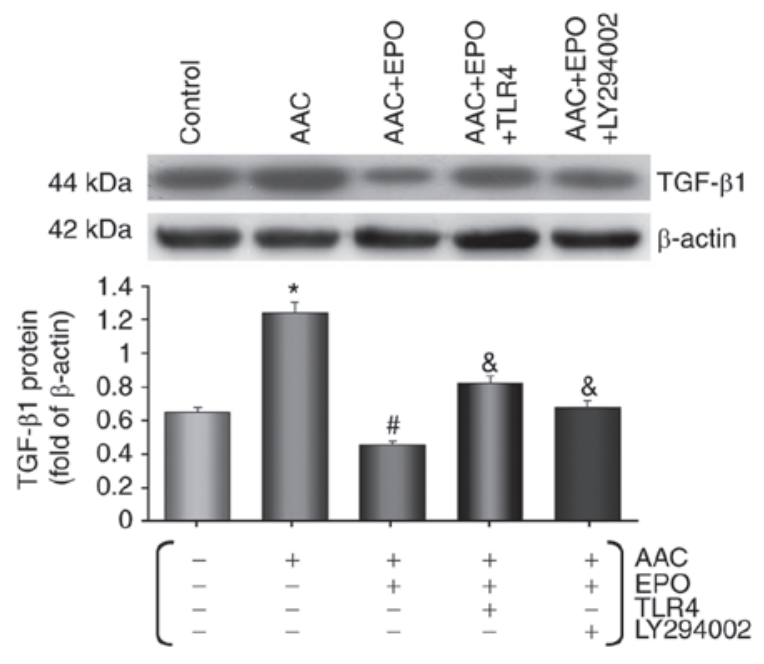

Figure 7. Effects of EPO overexpression on the protein expression levels of TGF- $\beta 1$ in hearts of rats subjected to AAC. Western blot analysis was performed to determine the protein expression levels of TGF- $\beta 1$. The data are expressed the mean \pm standard error of the mean ( $\mathrm{n}=6$ per group). ${ }^{*} \mathrm{P}<0.05$ vs. the control group; ${ }^{\#} \mathrm{P}<0.05$ vs. the $\mathrm{AAC}$ only group; and ${ }^{\circledR} \mathrm{P}<0.05$ vs. the $\mathrm{AAC}+\mathrm{EPO}$ group. $\mathrm{EPO}$, erythropoietin; TGF, transforming growth factor; AAC, abdominal aortic constriction; TLR4, Toll-like receptor 4.

EPO overexpression suppresses MMP-2 and MMP-9 expression. Since MMP-2 and MMP-9 are important components of fibrosis in the myocardium, their protein expression levels were examined by western blot analysis. As presented in Fig. 8, compared with the control, expression of MMP-9 and MMP-2 was significantly upregulated in the AAC only group $(\mathrm{P}<0.05)$, whereas they were downregulated in the $\mathrm{EPO}+\mathrm{AAC}$ group $(\mathrm{P}<0.05$ compared with the AAC only group). However, treatment with TLR4 or LY294002 attenuated the effects of EPO on MMP expression (Fig. 8).

\section{Discussion}

As a member of the pattern recognition receptors, TLRs were initially identified as Toll proteins for their roles in early embryogenesis of Drosophila melanogaster in 1984 (20). Human TLR4, secreted by the endoplasmic reticulum, was the first mammalian Toll protein to be characterized (21). TLR4 activates expression of several pro-inflammatory genes, chemokines, and cell surface molecules, and has many functions in various pathological conditions, including inflammatory bowel diseases, autoimmune disorders, infectious diseases, cardiovascular disease, neuronal degeneration, allergic diseases, or obesity-associated metabolic diseases (22). In previous studies, it has been suggested that there are close relationships between TLR4 and the fibrosis of organs, including liver (23), heart (24), kidney (25), and lung (26). In the present study, EPO was demonstrated to serve an antimyocardial fibrosis effect by downregulating the expression of TLR4. By contrast, forced overexpression of TLR4 attenuated the EPO-induced effects. A recent study has also reported that EPO improved necrotizing enterocolitis by reducing the expression levels of TLR4 and inflammatory mediators (27). The above-mentioned evidences indicate that TLR4 may have an important role in the beneficial effects of EPO on MF.
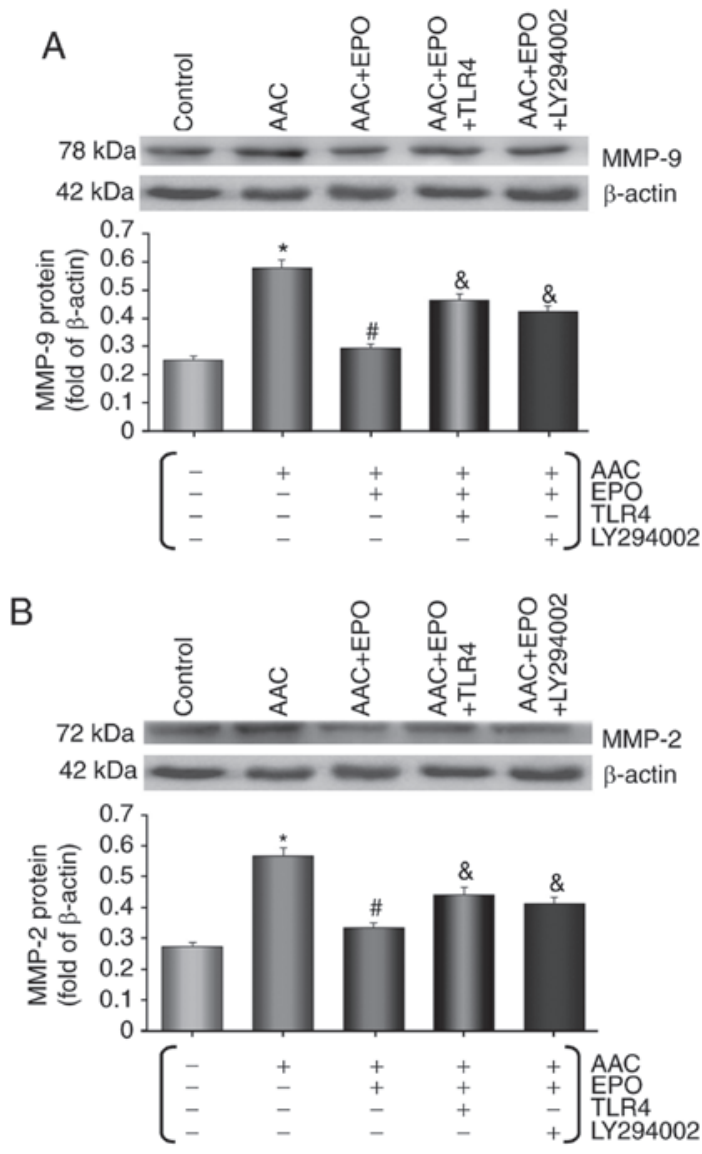

Figure 8. (A and B) Effects of EPO overexpression on the protein expression levels of MMP-9 and MMP-2 in the hearts of rats who underwent AAC. The protein expression levels of MMP-9 and MMP-2 were determined by western blot analysis. The data are expressed the mean \pm standard error of the mean $(n=6$ per group). ${ }^{*}<0.05$ vs. the control group; ${ }^{\#} \mathrm{P}<0.05$ vs. the AAC only group; and ${ }^{\&} \mathrm{P}<0.05$ vs. the AAC + EPO group. EPO, erythropoietin; MMP, matrix metalloproteinase; AAC, abdominal aortic constriction; TLR4, Toll-like receptor 4

Recent evidence has suggested that inflammation is one of the earliest processes in events of cardiac stress (28), and a link between inflammation and MF has been described. Inflammatory responses, triggered by tissue injury, promote tissue fibrosis via an TLR4-dependent signaling pathway (29). Downstream inflammatory mediators of the TLR4 signaling pathway, including TGF- $\beta 1$ (30), TNF- $\alpha$, IL-6 (31), IL-1 $\beta$, IL-17A (32), MMP-9 and MMP-2 (33), promote the occurrence and development of MF (22). In the present study, it was demonstrated that EPO significantly decreased the release of TGF- $\beta 1$, TNF- $\alpha$, IL- 6 , IL- $1 \beta$ and IL-17A in serum and inhibited the expression of MMP-9 and MMP-2 in myocardial tissue of MF in rats subjected to AAC, which was consistent with the findings presented in other reports $(11,34,35)$. The effects of EPO on inflammatory mediators may be key in attenuating MF. As aforementioned, the effect of EPO against MF may be through inhibiting the release of inflammatory mediators by downregulating the expression of TLR4. In fact, Pang et al (36) presented a similar mechanism, reporting that EPO had an anti-inflammatory effect in brain injury following acute carbon monoxide poisoning through TLR4 and nuclear factor- $\kappa \mathrm{B}$ signaling pathways.

Santhanam et al (37) summarized relevant studies from the past twenty years and demonstrated that the PI3K/Akt 
signaling pathway is a classic target for EPO to serve a protective role in cardiovascular and cerebrovascular disorders. In our previous studies, EPO was demonstrated to inhibit the proliferation of cardiac fibroblasts and cardiac hypertrophy via the PI3K/Akt signaling pathway (10). In the current study, EPO downregulated the expression of TLR4, as well as its downstream inflammatory mediators, by activating the PI3K/Akt signaling pathway. However, these effects were reversed when the PI3K/Akt signaling pathway was inhibited. Similarly, the study by Rong and Xijun (38) indicated that EPO pretreatment activated PI3K/Akt signaling to inhibit the expression and release of inflammatory mediators during ischemia-reperfusion of myocardial injury. In microglia, lithium suppressed lipopolysaccharide-induced TLR4 expression or microglial activation via the PI3K/Akt/FoxO1 signaling pathway (39). The controlled response between the PI3K/Akt signaling pathway and TLR4 has been also reported by Wang et al (40), who demonstrated that upregulation of miR-223 prevented the development of atherosclerosis by inhibiting TLR4 signaling via activation of the PI3K/Akt pathway.

Taken together, the present study demonstrated that EPO had a protective role against MF by activating the PI3K/Akt signaling pathway and by downregulating TLR4 expression, thereby inhibiting the release of TGF- $\beta 1$, TNF- $\alpha$, IL-6, IL-1 $\beta$, IL-17A, MMP-9 and MMP-2. Thus, the PI3K/Akt/TLR4 signaling pathway may be a common signaling pathway of the EPO-mediated anti-inflammatory activity that results in attenuating MF. A limitation of the present study is that left ventricle hypertrophy was evaluated only through LVWI and pathology analysis. However, the present findings give valuable information on the protective role of EPO in MF through regulation of TLR4.

\section{Acknowledgements}

Not applicable.

\section{Funding}

This study was supported by the Natural Scientific Foundation of China (grant no. 81260019) and the Natural Scientific Foundation of the Jiangxi province (grant no. 2010GQY0334).

\section{Availability of data and materials}

The analyzed datasets generated during the study are available from the corresponding author on reasonable request.

\section{Authors' contributions}

FL was in charge of molecular biology experiments. YW performed the histological examination and was a major contributor in writing the manuscript. JK was in charge of animal experiments. MW assisted animal experiments. ZZ and JP assisted with molecular biology experiments.

\section{Ethics approval and consent to participate}

Experiments involving animals were approved by the Experimental Animal Ethics Committee of the Nanchang University (Nanchang, China; approval no. 2015050612).

\section{Consent for publication}

Not applicable.

\section{Competing interests}

The authors declare that they have no competing interests.

\section{References}

1. Travers JG, Kamal FA, Robbins J, Yutzey KE and Blaxall BC: Cardiac fibrosis: The fibroblast awakens. Circ Res 118: 1021-1040, 2016.

2. Moore-Morris T, Guimarães-Camboa N, Yutzey KE, Pucéat M and Evans SM: Cardiac fibroblasts: From development to heart failure. J Mol Med (Berl) 93: 823-830, 2015.

3. Lombardero M, Kovacs K and Scheithauer BW: Erythropoietin: A hormone with multiple functions. Pathobiology 78: 41-53, 2011.

4. Ma BX, Li J, Li H and Wu SS: Recombinant human erythropoietin protects myocardial cells from apoptosis via the janus-activated kinase 2/signal transducer and activator of transcription 5 pathway in rats with epilepsy. Curr Ther Res Clin Exp 77: 90-98, 2015.

5. Elshiekh M, Kadkhodaee M, Seifi B, Ranjbaran M and Ahghari P: Ameliorative effect of recombinant human erythropoietin and ischemic preconditioning on renal ischemia reperfusion injury in rats. Nephrourol Mon 7: e31152, 2015.

6. Noguchi T, Ohta S, Kakinoki R, Ikeguchi R, Kaizawa Y, Oda H and Matsuda S: The neuroprotective effect of erythropoietin on spinal motor neurons after nerve root avulsion injury in rats. Restor Neurol Neurosci 33: 461-470, 2015.

7. Nakamura S, Sho M, Koyama F, Ueda T, Nishigori N, Inoue T, Nakamoto T, Fujii H, Yoshikawa S, Inatsugi N and Nakajima Y: Erythropoietin attenuates intestinal inflammation and promotes tissue regeneration. Scand J Gastroenterol 50: 1094-1102, 2015.

8. Geng XC, Hu ZP and Lian GY: Erythropoietin ameliorates renal interstitial fibrosis via the inhibition of fibrocyte accumulation. Mol Med Rep 11: 3860-3865, 2015.

9. Park SY, Lee JY, Tak WY, Kweon YO and Lee MS: Erythropoietin decreases carbon tetrachloride-induced hepatic fibrosis by inhibiting transforming growth factor-beta. Chin Med J (Engl) 125: 3098-3103, 2012.

10. Zhang XJ, Ma YX, Wen Y and Xu XJ: Erythropoietin suppresses the expressions of TGF-betal and collagen in rat cardiac fibroblasts induced by angiotensin II. Zhonghua Xin Xue Guan Bing Za Zhi 36: 636-640, 2008 (In Chinese).

11. Wang LP, Yang XH, Wang XJ, Li SM, Sun N and Zhang T: Erythropoietin decreases the occurrence of myocardial fibrosis by inhibiting the NADPH-ERK-NF-x03BA;B pathway. Cardiology 133: 97-108, 2016.

12. Tada H, Kagaya Y, Takeda M, Ohta J, Asaumi Y, Satoh K, Ito K, Karibe A, Shirato K, Minegishi N and Shimokawa H: Endogenous erythropoietin system in non-hematopoietic lineage cells plays a protective role in myocardial ischemia/reperfusion. Cardiovasc Res 71: 466-477, 2006.

13. Jun JH, Jun NH, Shim JK, Shin EJ and Kwak YL: Erythropoietin protects myocardium against ischemia-reperfusion injury under moderate hyperglycemia. Eur J Pharmacol 745: 1-9, 2014.

14. Lu J, Yao YY, Dai QM, Ma GS, Zhang SF, Cao L, Ren LQ and Liu NF: Erythropoietin attenuates cardiac dysfunction by increasing myocardial angiogenesis and inhibiting interstitial fibrosis in diabetic rats. Cardiovasc Diabetol 11: 105, 2012.

15. Liu S, Zhao C, Yang C, Li X, Huang H, Liu N, Li S, Wang X and Liu J: Gambogic acid suppresses pressure overload cardiac hypertrophy in rats. Am J Cardiovasc Dis 3: 227-238, 2013.

16. Merkulov S, Chen X, Chandler MP and Stelzer JE: In vivo cardiac myosin binding protein $\mathrm{C}$ gene transfer rescues myofilament contractile dysfunction in cardiac myosin binding protein $\mathrm{C}$ null mice. Circ Heart Fail 5: 635-644, 2012.

17. Wei XL, Fang RT, Yang YH, Bi XY, Ren GX, Luo AL, Zhao M and Zang WJ: Protective effects of extracts from Pomegranate peels and seeds on liver fibrosisinduced by carbon tetrachloride in rats. BMC Complement Altern Med 15: 389, 2015.

18. Livak KJ and Schmittgen TD: Analysis of relative gene expression data using real-time quantitative PCR and the 2(-delta delta $\mathrm{C}(\mathrm{T})$ ) method. Methods 25: 402-408, 2001. 
19. Chen CJ, He H, Luo Y, Zhou M, Yin D and He M: Involvement of Bcl-2 Signal pathway in the protective effects of apigenin on anoxia/reoxygenation-induced myocardium injury. J Cardiovasc Pharmacol 67: 152-163, 2016.

20. Steward R, McNally FJ and Schedl P: Isolation of the dorsal locus of Drosophila. Nature 311: 262-265; 1984

21. Medzhitov R, Preston-Hurlburt P and Janeway CA Jr: A human homologue of the Drosophila Toll protein signals activation of adaptive immunity. Nature 388: 394-397, 1997.

22. Yang Y, Lv J, Jiang S, Ma Z, Wang D, Hu W, Deng C, Fan C, Di S, Sun Y and Yi W: The emerging role of Toll-like receptor 4 in myocardial inflammation. Cell Death Dis 7: e2234, 2016.

23. Zhao HW, Zhang ZF, Chai X, Li GQ, Cui HR, Wang HB, Meng YK, Liu HM, Wang JB, Li RS, et al: Oxymatrine attenuates $\mathrm{CCl} 4$-induced hepatic fibrosis via modulation of TLR4-dependent inflammatory and TGF- $\beta 1$ signaling pathways. Int Immunopharmacol 36: 249-255, 2016.

24. Zhang Y, Peng W, Ao X, Dai H, Yuan L, Huang X and Zhou Q: TAK-242, a Toll-like receptor 4 antagonist, protects against aldosterone-induced cardiac and renal injury. PLoS One 10: e0142456, 2015.

25. Ma ZJ, Zhang XN, Li L, Yang W, Wang SS, Guo X, Sun P and Chen LM: Tripterygium glycosides tablet ameliorates renal tubulointerstitial fibrosis via the Toll-like receptor 4/nuclear factor kappa B signaling pathway in high-fat diet fed and streptozotocin-induced diabetic rats. J Diabetes Res 2015: 390428, 2015

26. Yang HZ, Wang JP, Mi S, Liu HZ, Cui B, Yan HM, Yan J, Li Z, Liu $\mathrm{H}$, Hua F, et al: TLR4 activity is required in the resolution of pulmonary inflammation and fibrosis after acute and chronic lung injury. Am J Pathol 180: 275-292, 2012.

27. Qi W, Shen Q, Zhang L, Han LP and Wang S: Study on the inflammatory intervention of erythropoietin on NEC. Exp Ther Med 11: 2221-2224, 2016.

28. Glezeva N and Baugh JA: Role of inflammation in the pathogenesis of heart failure with preserved ejection fraction and its potential as a therapeutic target. Heart Fail Rev 19: 681-694, 2014

29. Zhang W, Lavine KJ, Epelman S, Evans SA, Weinheimer CJ, Barger PM and Mann DL: Necrotic myocardial cells release damage-associated molecular patterns that provoke fibroblast activation in vitro and trigger myocardial inflammation and fibrosis in vivo. J Am Heart Assoc 4: e001993, 2015.

30. Stifano G, Affandi AJ, Mathes AL, Rice LM, Nakerakanti S, Nazari B, Lee J, Christmann RB and Lafyatis R: Chronic Toll-like receptor 4 stimulation in skin induces inflammation, macrophage activation, transforming growth factor beta signature gene expression, and fibrosis. Arthritis Res Ther 16: R136, 2014.

31. Zhang Y, Wang JH, Zhang YY, Wang YZ, Wang J, Zhao Y, Jin XX, Xue GL, Li PH, Sun YL, et al: Deletion of interleukin-6 alleviated interstitial fibrosis in streptozotocin-induced diabetic cardiomyopathy of mice through affecting TGF $\beta 1$ and miR-29 pathways. Sci Rep 6: 23010, 2016.
32. Zhou SF, Yuan J, Liao MY, Xia N, Tang TT, Li JJ, Jiao J, Dong WY, Nie SF, Zhu ZF, et al: IL-17A promotes ventricular remodeling after myocardial infarction. J Mol Med (Berl) 92: 1105-1116, 2014.

33. Pulskens WP, Rampanelli E, Teske GJ, Butter LM, Claessen N, Luirink IK, van der Poll T, Florquin S and Leemans JC: TLR4 promotes fibrosis but attenuates tubular damage in progressive renal injury. J Am Soc Nephrol 21: 1299-1308, 2010.

34. Turhan AH, Atici A, Muşlu N, Polat A and Sungur MA: Erythropoietin may attenuate lung inflammation in a rat model of meconium aspiration syndrome. Exp Lung Res 42: 199-204, 2016.

35. Sifringer M, Genz K, Brait D, Brehmer F, Löber R, Weichelt U, Kaindl AM, Gerstner B and Felderhoff-Mueser U: Erythropoietin attenuates hyperoxia-induced cell death by modulation of inflammatory mediators and matrix metalloproteinases. Dev Neurosci 31: 394-402, 2009.

36. Pang L, Zhang N, Dong N, Wang DW, Xu DH, Zhang P and Meng XW: Erythropoietin protects rat brain injury from carbon monoxide poisoning by inhibiting Toll-like receptor 4/NF-kappa B-dependent inflammatory responses. Inflammation 39: 561-568, 2016.

37. Santhanam AV, d'Uscio LV and Katusic ZS: Cardiovascular effects of erythropoietin an update. Adv Pharmacol 60: 257-285, 2010.

38. Rong R and Xijun X: Erythropoietin pretreatment suppresses inflammation by activating the PI3K/Akt signaling pathway in myocardial ischemia-reperfusion injury. Exp Ther Med 10: 413-418, 2015.

39. Dong H, Zhang X, Dai X, Lu S, Gui B, Jin W, Zhang S, Zhang S and Qian Y: Lithium ameliorates lipopolysaccharide-induced microglial activation via inhibition of Toll-like receptor 4 expression by activating the PI3K/Akt/FoxO1 pathway. J Neuroinflammation 14: 140-144, 2014.

40. Wang J, Bai X, Song Q, Fan F, Hu Z, Cheng G and Zhang Y: mir-223 inhibits lipid deposition and inflammation by suppressing Toll-like receptor 4 signaling in macrophages. Int J Mol Sci 16: 24965-24982, 2015

This work is licensed under a Creative Commons Attribution-NonCommercial-NoDerivatives 4.0 International (CC BY-NC-ND 4.0) License. 